\title{
A closer look at the functional diversity of cytotoxins from Southeast Asian cobras: Biomedical and clinical significance
}

\section{Choo Hock Tan ${ }^{1 *}$, Ho Phin Chong ${ }^{1}$, Kae Yi Tan ${ }^{2}$}

\begin{abstract}
${ }^{1}$ Venom Research and Toxicology Lab, Department of Pharmacology, Faculty of Medicine, University of Malaya, Kuala Lumpur, Malaysia 2 Protein and Interactomics Lab, Department of Molecular Medicine, Faculty of Medicine, University of Malaya, Kuala Lumpur, Malaysia *Email: tanch@um.edu.my
\end{abstract}

\section{Introduction}

Cobra venoms contain cytotoxic components that can be further explored for anticancer potential. Cobra venom cytotoxins
are polypeptides of $60-70$ amino acid residues held by four disulfide crosslinks, forming a three protruding fingers-like
structure and is grouped under the three-finger toxin superfamily. Its unique structure gives rise to its diverse
pharmacological actions such as pore formation and disruption of cell membranes. Its cytotoxic properties, nevertheless,
imply the possibility to harness cobra cytotoxins in the development of new anticancer drugs. The present study explores
the two most common cobra species in Southeast Asia, Naja sumatrana (equatorial spitting cobra) and Naja kaouthia
(monocled cobra) to elucidate the prospect of cytotoxin-derived anticancer therapeutics, through investigating the
anticancer properties of the purified cytotoxins of $N$. sumatrana and $N$. kaouthia in breast, prostate, and lung cancer cell
lines. The study further delved into the specificity and selectivity of the cytotoxins and verified the cell death mechanisms
underlying their cytotoxic activities.

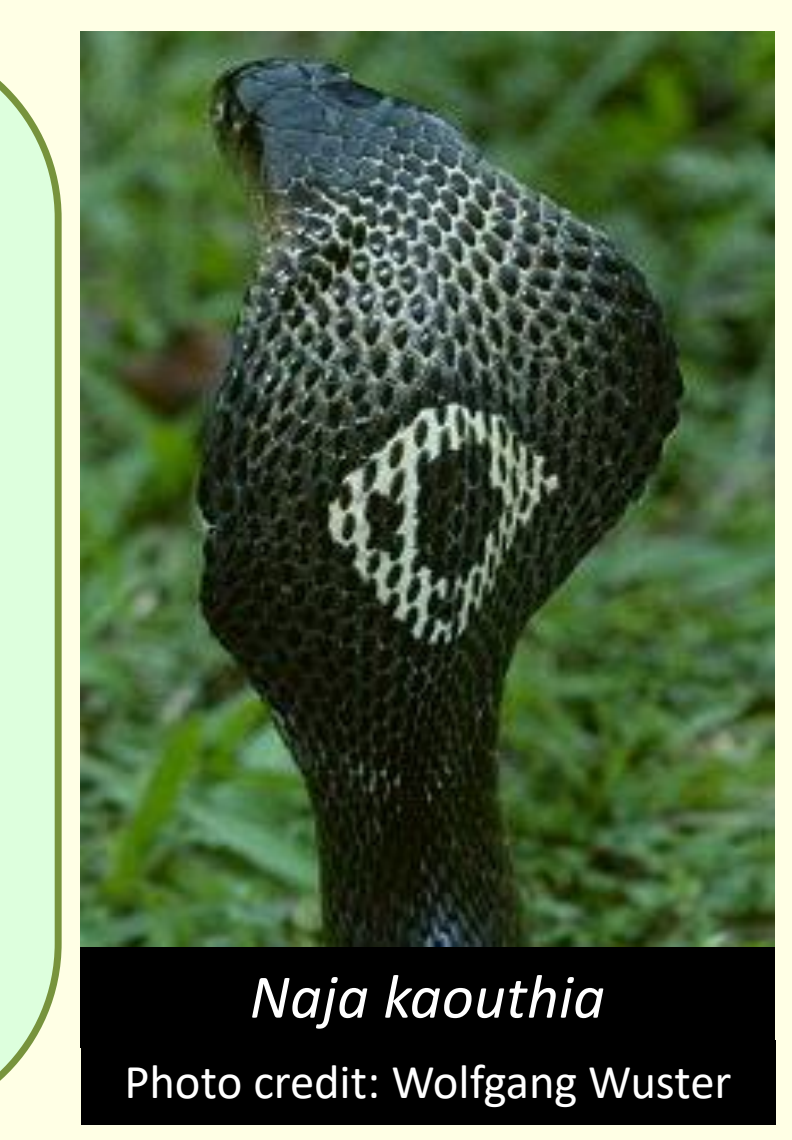

\section{Methods}

\section{Fractionation \& Purification}

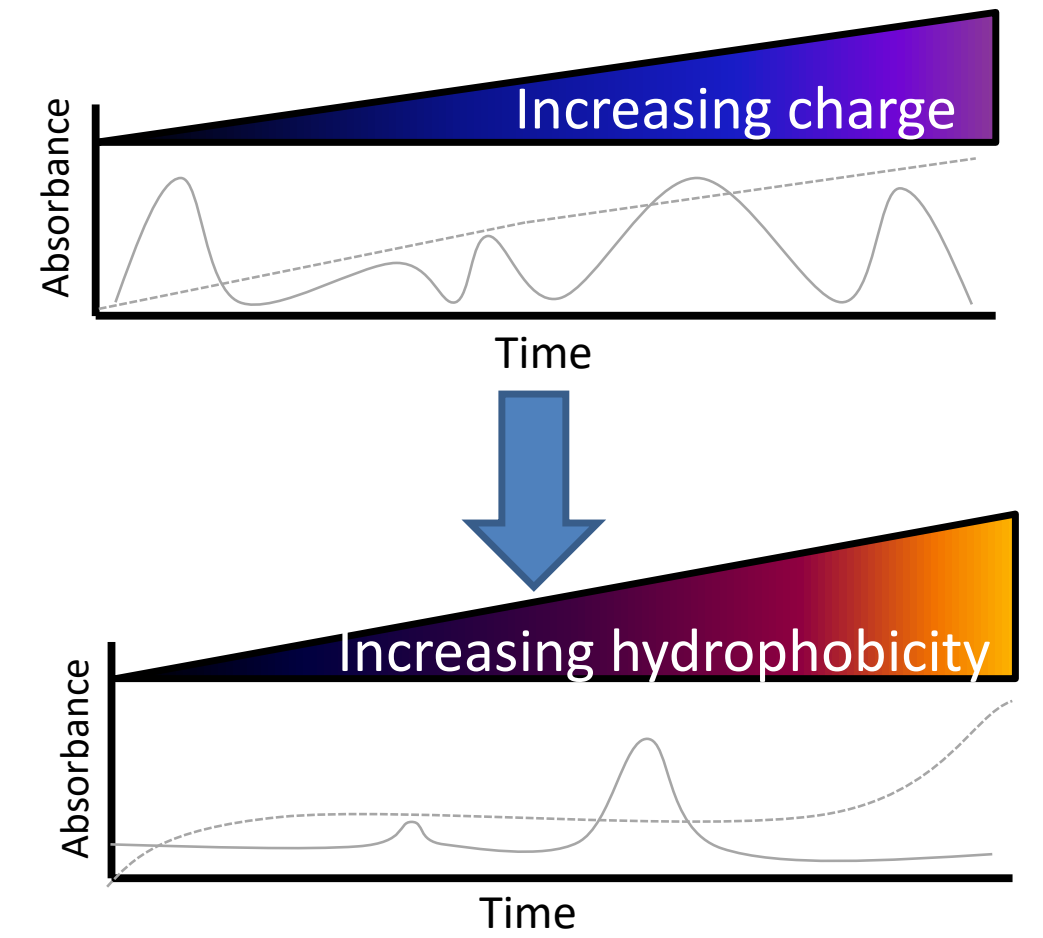

Sequential fractionation with cation exchange chromatography followed by reverse-phase high performance liquid chromatography

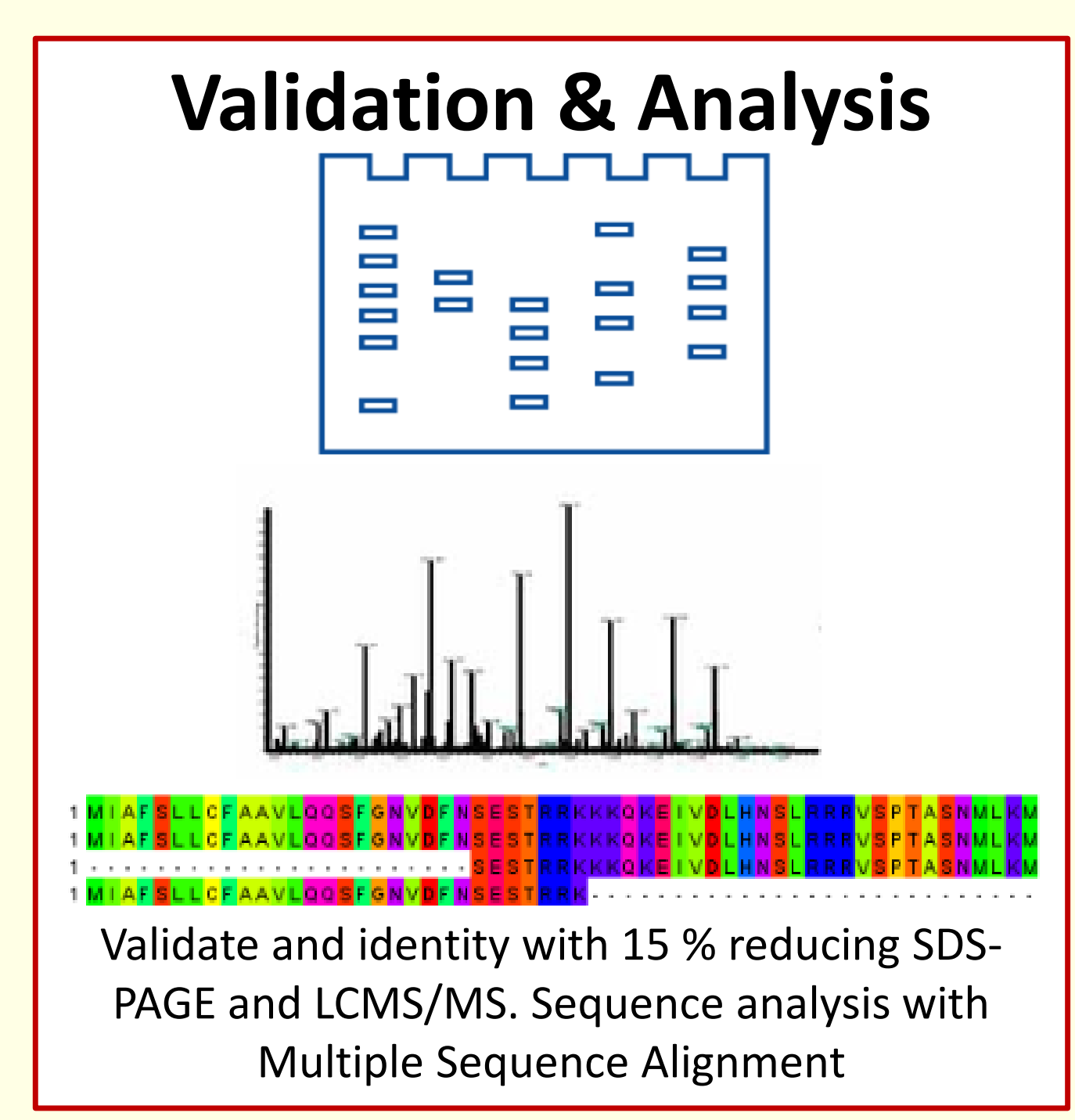

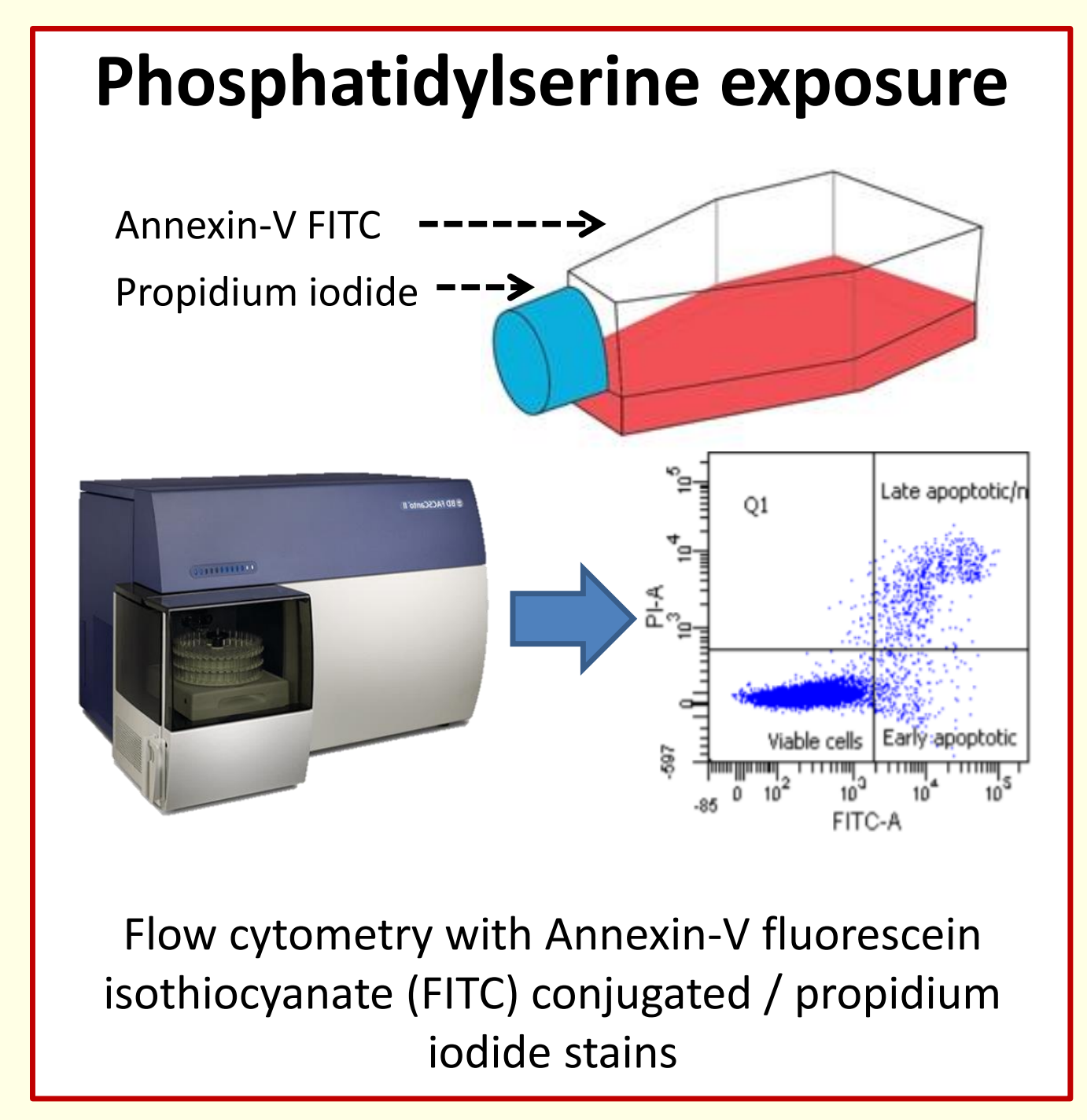

Cytotoxicity tested with MTT on A549, PC-3 and MCF-7 cell lines. 5-fluorouracil used as positive control.

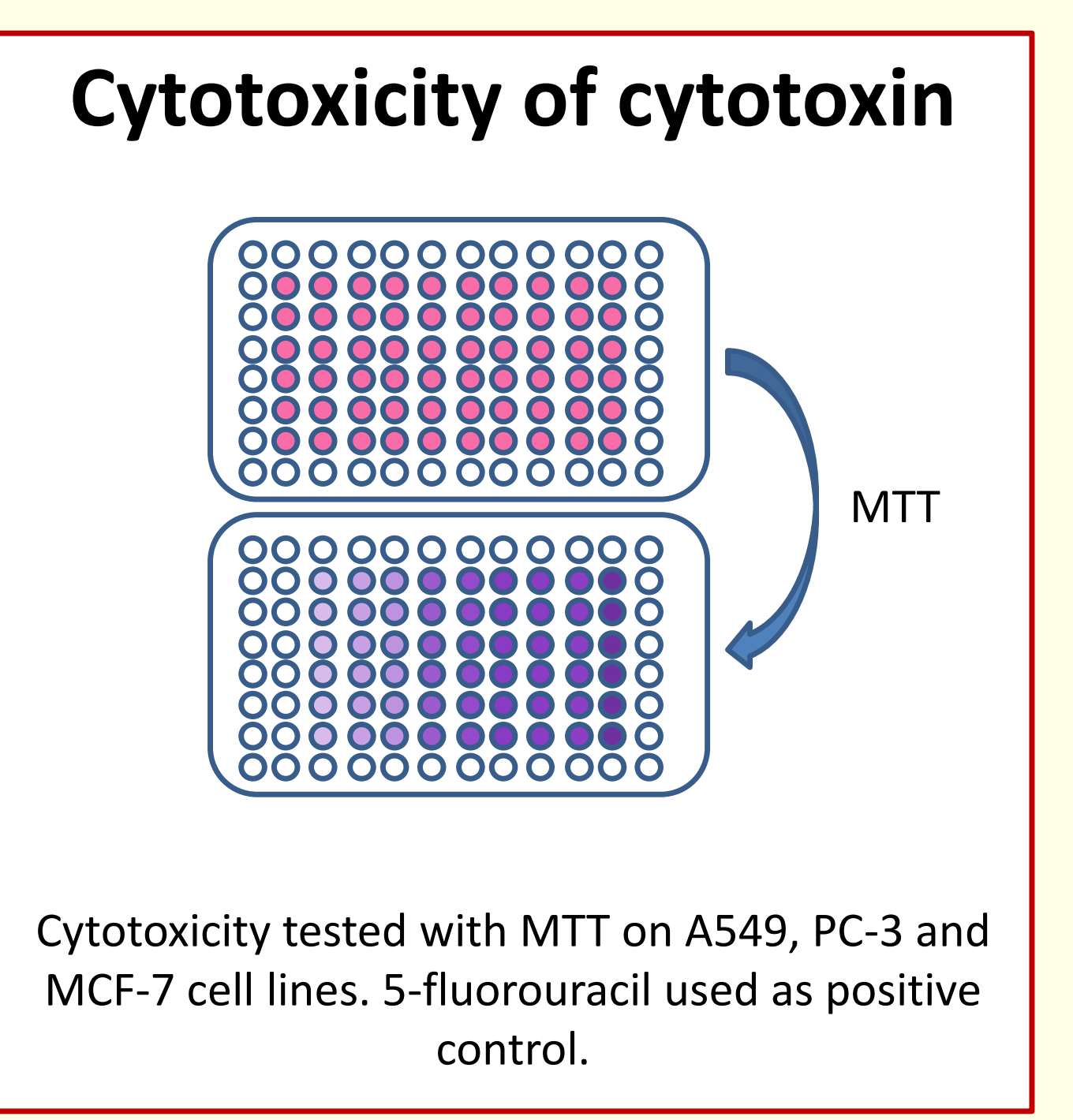

\section{Results}

\section{Purification of cytotoxins}

吗

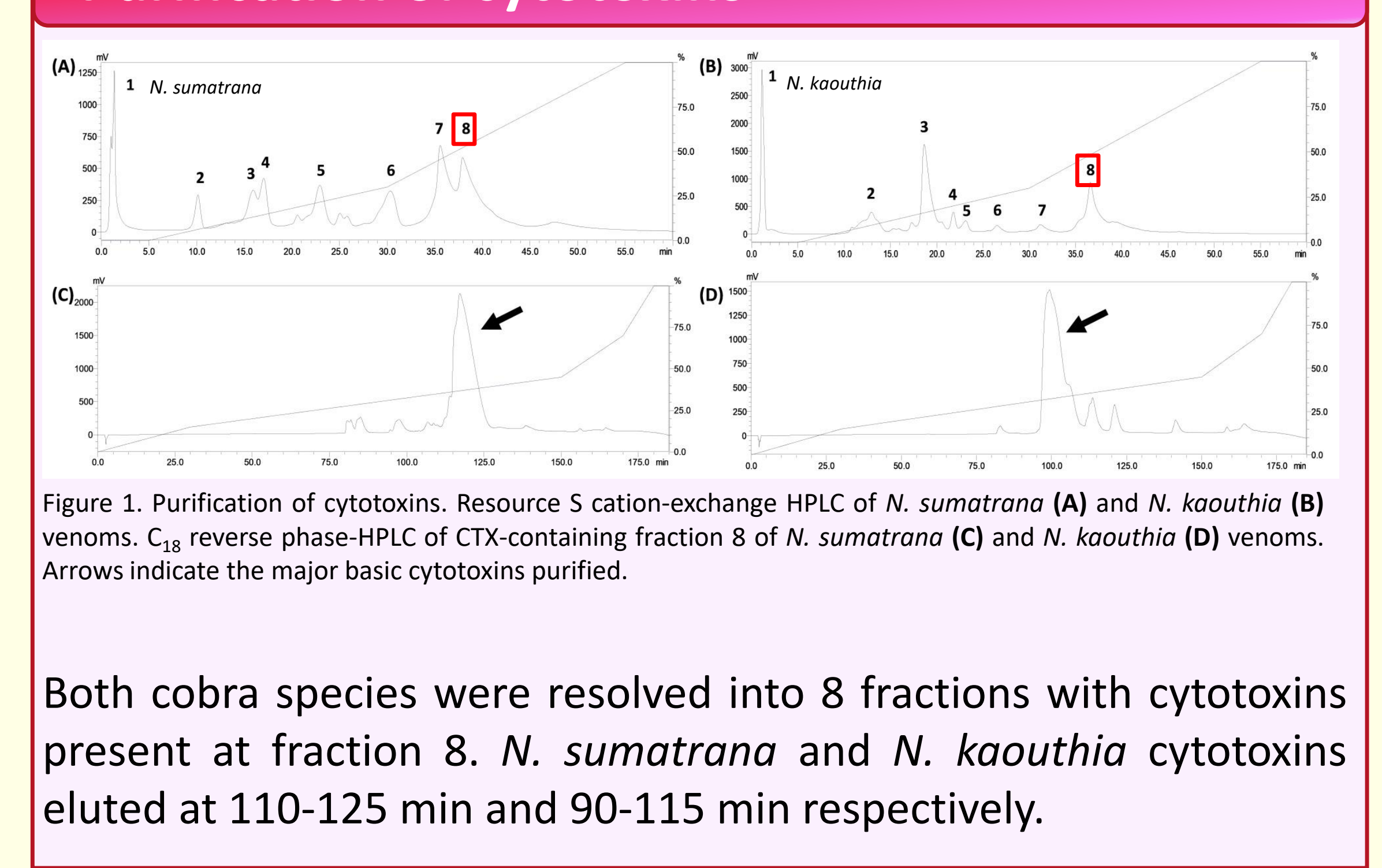

\section{Ce
(A)
(B)}
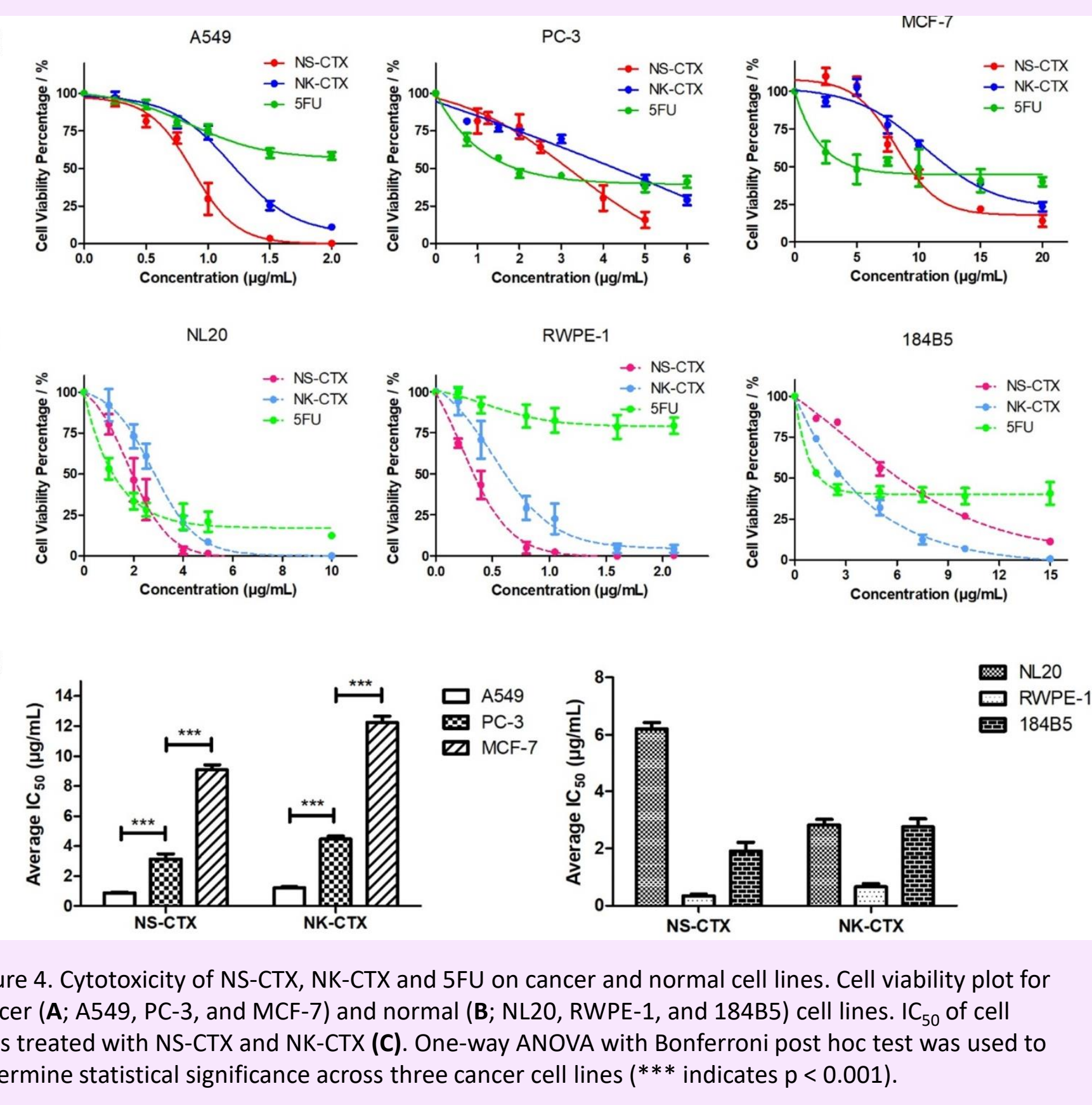

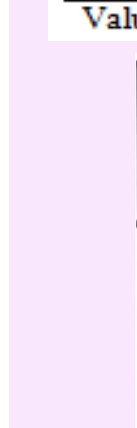

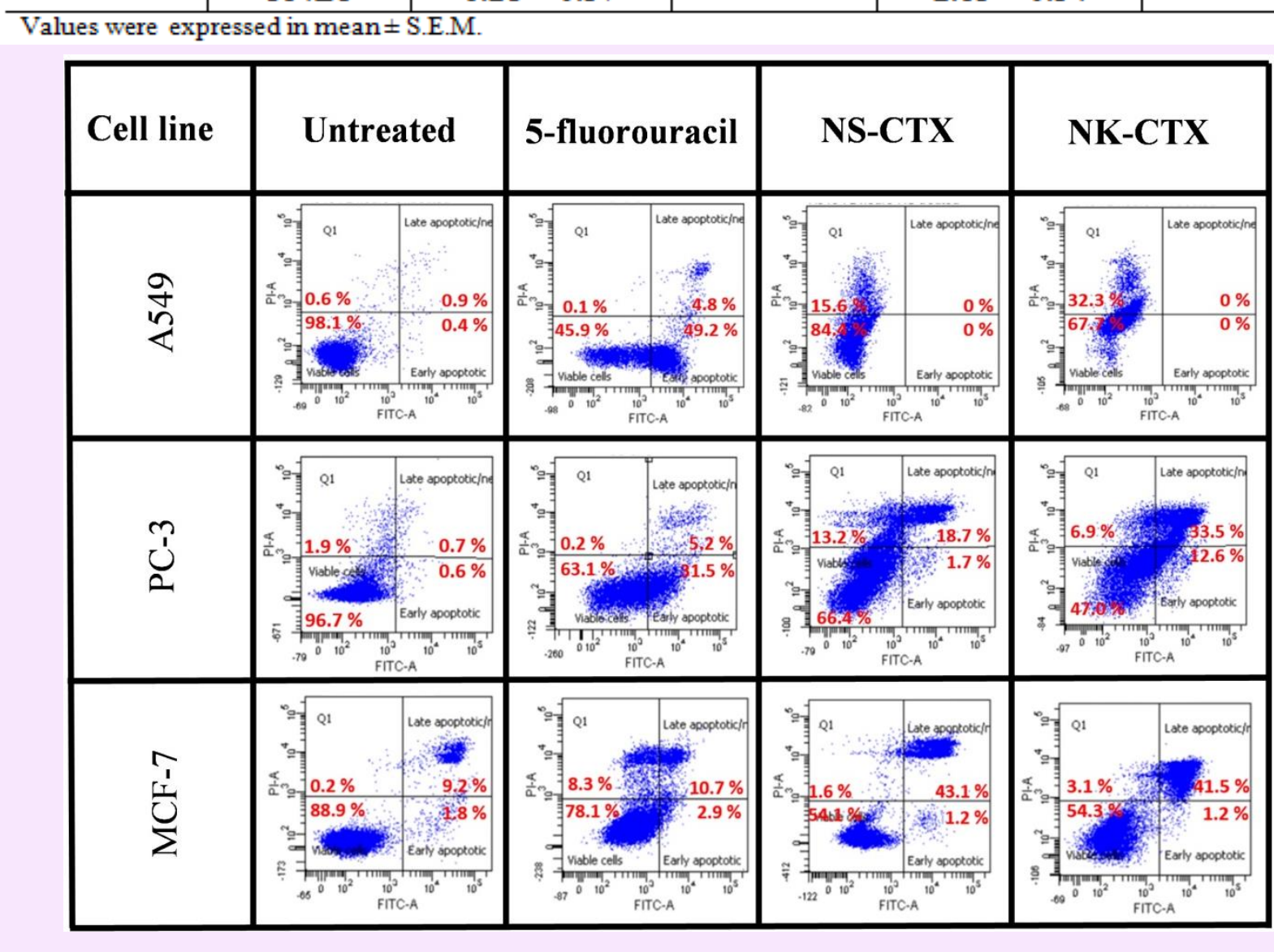

Figure 5. Dot plots showing flow cytometrit
induced by NS-CT and NK-CT treatment.

NS-CTX, NK-CTX, and 5FU showed dose-dependent cytotoxic activities in cancer cell lines and norma cell lines. Increasing cytotoxicity in the following order: MCF-7 < PC-3 < A549. Normal breast and prostate cells were more susceptible to NS-CTX and NK-CTX, whilst selectivity was seen in lung cell lines. Flow cytometry demonstrated varying cell death mechanisms, Predominantly necrosis and late apoptosis in the cancer cell lines. Necrosis in the A549 and mainly late apoptosis in the MCF-7 cell line.

\section{Validation and sequence analysis of cytotoxins}
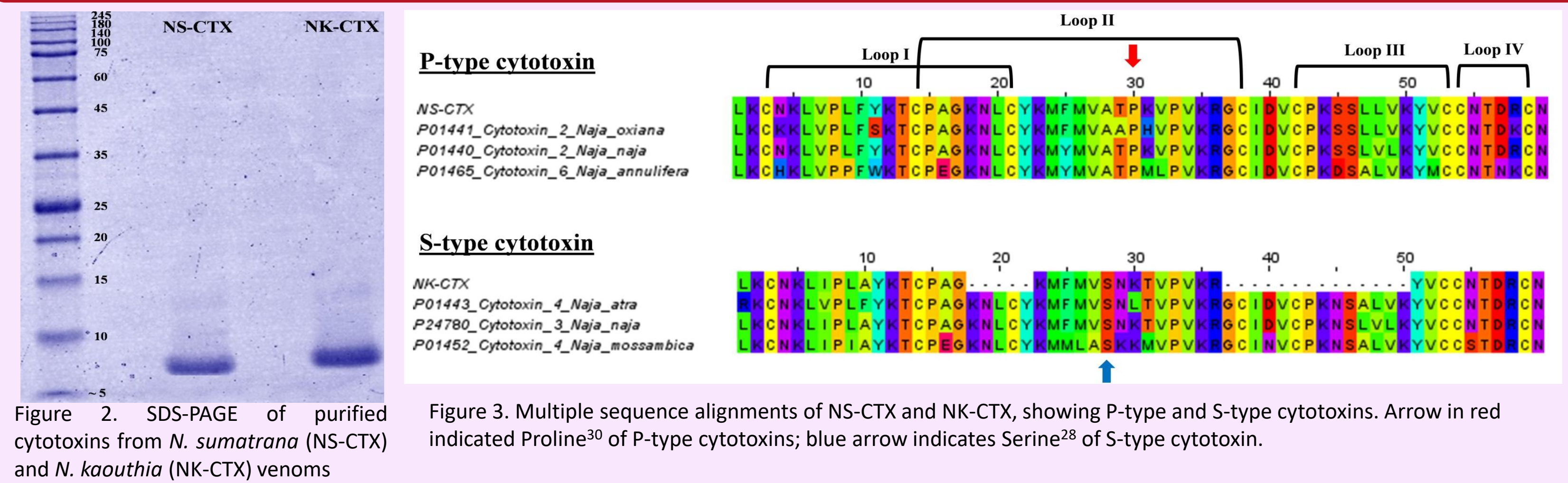

S-type cytotoxin
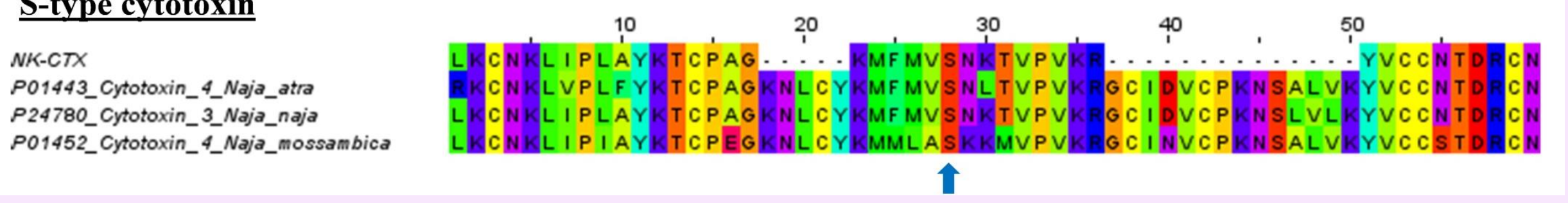

SDS-PAGE showed homogenous protein band of approximately $7 \mathrm{kDa}$ for both purified cytotoxins. Multiple sequence alignment showed the presence of Pro ${ }^{30}$ in NS-CTX whilst NK-CTX possessing $\mathrm{Ser}^{28}$, respectively categorizing the cytotoxins are P-type and S-type cytotoxins. 